\title{
Qualité de la viande de dromadaire dans les abattoirs de Ouargla en Algérie. I. Quelques caractéristiques physico-chimiques de la viande au cours de la maturation
}

\author{
A. Benaissa ${ }^{1} *$ A. Ould El Hadj-Khelil ${ }^{1}$ A. Adamou ${ }^{1}$ \\ B. Babelhadj ${ }^{1}$ M. Mehirig ${ }^{1}$ B. Boufaghes ${ }^{1}$ \\ M. Attoussi ${ }^{1}$ H. Samili ${ }^{2}$ S. Becila ${ }^{2}$
}

\section{Mots-clés}

Camelus dromedarius - Viande de chameau - Muscle - Maturation des produits - Propriété physicochimique - $\mathrm{pH}$ - Algérie.

\begin{abstract}
Résumé
Les paramètres physico-chimiques au cours de la maturation de quatre muscles (semitendinosus [ST], rectus femoris [RF], longissimus thoracis [LT] et semimembranosus $[\mathrm{SM}]$ ), conservés à $4{ }^{\circ} \mathrm{C}$, ont été mesurés chez des dromadaires mâles ( 10 jeunes de trois à quatre ans et 10 adultes de plus de cinq ans) de race Sahraoui. Une diminution de la température post mortem a atteint une valeur minimale de $5,7 \pm 0,20{ }^{\circ} \mathrm{C}$ (LT) chez les adultes, alors que la valeur minimale enregistrée chez les jeunes a été plus basse avec 3,9 $\pm 0,68{ }^{\circ} \mathrm{C}(\mathrm{ST})$. Le $\mathrm{pH}$ des muscles étudiés a diminué au cours de la période post mortem. Les valeurs minimales enregistrées ont varié de 5,2 $\pm 1,11$ (RF) à 5,7 $\pm 0,02(\mathrm{LT})$ chez les chamelons, et de 5,6 $\pm 0,05(\mathrm{SM})$ à 6,3 $\pm 0,53$ (ST) chez les adultes. La quantité d'eau extractible a augmenté pour les quatre muscles et pour les deux groupes d'âge. Les valeurs maximales obtenues ont été de 0,21 $\pm 0,040 \mathrm{~g}$ par gramme de muscle RF jeune et de 0,21 $\pm 0,009 \mathrm{~g}$ par gramme de muscle RF adulte. Cela signifie que la capacité de rétention d'eau des différents muscles a diminué au cours du temps post mortem. La conductivité électrique n'a pas suivi le même profil pour tous les muscles étudiés. Une légère augmentation de ce paramètre dans le temps a été perceptible pour le muscle LT $(117,3 \pm 0,30 \mu \mathrm{S} / \mathrm{cm} / \mathrm{g})$ chez les jeunes, et ST $(158,3$ $\pm 0,14 \mu \mathrm{S} / \mathrm{cm} / \mathrm{g}$ ) chez les adultes.
\end{abstract}

\section{INTRODUCTION}

La viande, première source de protéines animales, se situe, de par sa richesse en acides aminés indispensables, parmi les protéines nobles (10). Le dromadaire, avec son bon rendement carcasse et à la qualité diététique de sa viande, est apprécié et consommé à grande échelle dans le Sahara algérien (19), et dans l'ensemble du monde arabo-musulman (9). Parmi les qualités recherchées,

\footnotetext{
1. Laboratoire de protection des écosystèmes des zones arides et semi-arides, Université Kasdi Merbah, Ouargla 30000, Algérie.

2. Laboratoire de biotechnologie et qualité des aliments, Institut de la nutrition, de l'alimentation et des technologies agroalimentaires, Université de Constantine 1, Algérie.

* Auteur pour la correspondance

Tél. : 772213516 ; e-mail : benaissaatika@gmail.com
}

la qualité organoleptique s'apprécie essentiellement au travers de différents critères qui sont : la couleur, la flaveur, la jutosité et la tendreté (11).

Cette tendreté, perçue par le consommateur comme la qualité organoleptique la plus importante, a fait l'objet de recherches pour tenter de mieux comprendre et contrôler cette caractéristique sensorielle $(14,17)$. Cependant, les variations individuelles sont très importantes. Picard et coll. (20) rapportent que chez les animaux producteurs de viande, la qualité de la viande varie en fonction de facteurs non seulement biologiques mais aussi technologiques, aussi bien ante mortem que post mortem.

L'insatisfaction que ressent le consommateur trouve ses sources dans la difficulté à caractériser de façon objective, et donc à mesurer l'ensemble des paramètres qui définissent la tendreté (6). En effet, après l'abattage, la structure myofibrillaire des muscles subit de profondes 
modifications qui dépendent en grande partie des caractéristiques enzymatiques et physico-chimiques des fibres. Ces dernières mettent en jeu, entre autres, la diminution du $\mathrm{pH}$, de la température et de la capacité de rétention d'eau. La vitesse et l'intensité de ces paramètres conditionnent étroitement la dichotomie dureté/tendreté. En outre, dès la mort de l'animal, d'autres processus d'attendrissage se mettent en place. Ils sont dus à la protéolyse myofibrillaire. Cette protéolyse cause la rupture des myofibrilles et conduit à un attendrissage de la viande (13). Seule une bonne compréhension de ces mécanismes permet de contrôler la dureté finale de la viande. Afin d'apporter une contribution à l'étude de ces mécanismes, un travail expérimental a été mené sur quelques muscles de dromadaires.

\section{MATERIEL ET METHODES}

\section{Matériel biologique}

La viande de dromadaire étudiée provenait d'animaux abattus à l'abattoir de Ouargla en Algérie. Les animaux étaient des mâles de race Sahraoui, de différents âges (10 adultes de plus de cinq ans et 10 jeunes de trois à quatre ans). Les muscles étudiés ont été : le semitendinosus (ST), le rectus femoris (RF), le longissimus thoracis (LT) et le semimembranosus (SM). Les muscles ont été prélevés après la dépouille et l'éviscération des carcasses. Les paramètres ont été étudiés en cinétique à 2, 4, 6, 8, 24 et 48 heures post mortem. Pour chacune des heures, les mesures ont été répétées trois fois pour tous les paramètres.

\section{Méthodes d'analyses}

\section{Température}

La température intramusculaire $(\mathrm{T})$ des différents échantillons a été directement lue à partir d'un thermomètre électronique de modèle Testo 206 équipé d'une sonde pénétrante permettant la mesure de pour chaque échantillon et la température relevée a été la moyenne des trois lectures réalisées.

\section{$p H$}

Le $\mathrm{pH}$ a été directement lu à partir d'un $\mathrm{pH}$ mètre électronique de modèle Testo 206, équipé d'une sonde pénétrante permettant la mesure du $\mathrm{pH}$ au cœur du muscle. La valeur du $\mathrm{pH}$ a été la moyenne de trois essais pour chaque muscle.

\section{Capacité de rétention d'eau}

La quantité de jus extractible (QJE) a été déterminée à partir de trois grammes de viande broyés pendant environ $8 \mathrm{~s}$ et centrifugé à $5000 \mathrm{~g}$ pendant $90 \mathrm{~min}$ à l'aide d'une centrifugeuse de type Rotina $380 \mathrm{~A}$ à $4{ }^{\circ} \mathrm{C}$. La capacité de rétention d'eau a été estimée par la quantité de jus relarguée en gramme par gramme de muscle (23, 27). Trois essais ont été réalisés pour chaque muscle : la capacité de rétention d'eau tissulaire a été la moyenne des trois mesures.

\section{Conductivité électrique}

La conductivité électrique (CE) a été lue directement sur le conductivimètre WTW modèle 315i (Weilheim, Allemagne) à $25^{\circ} \mathrm{C}$. La $\mathrm{CE}$ a ensuite été déterminée sur du jus extrait d'un gramme de muscle.

\section{Analyse statistique}

Les analyses statistiques ont inclus deux étapes : l'analyse de la variabilité entre les paramètres mesurés dans les quatre muscles chez les animaux de même tranche d'âge, jeune (j) ou adulte (a), puis l'analyse comparative pour déterminer la variabilité des paramètres physico-chimiques des muscles. L'analyse de la variabilité interne a inclus la description de la moyenne pour chacun des paramètres mesurés et les corrélations entre les différents paramètres mesurés pour chaque muscle (corrélation de Pearson).

\section{RESULTATS}

\section{Paramètres physico-chimiques des muscles jeunes}

La température n'a pas été significativement différente entre les muscles des adultes (tableau I). Le pH et la QJE n'ont pas non plus montré de différence significative. La conductivité électrique a été en revanche significativement plus élevée dans les muscles ST et LT que dans les muscles SM et RF.

\section{Paramètres physico-chimiques des muscles adultes}

La température n'a pas été significativement différente entre les muscles des adultes mais les valeurs numériques ont été plus élevées pour SM et LT, comme c'était le cas dans les muscles jeunes (tableau II). Le $\mathrm{pH}$ a été comparable dans tous les muscles. Les différences de la QJE n'ont pas été significatives mais la valeur la plus forte a été notée dans le muscle RF, alors qu'il avait la valeur la plus faible chez le jeune. La conductivité électrique a été, en revanche, significativement plus élevée dans le muscle ST que dans les muscles SM, LT et RF.

\section{Tableau I}

Moyenne \pm écart-type des paramètres physicochimiques des muscles étudiés jeunes (j)

\begin{tabular}{|c|c|c|c|c|}
\hline & STj & SMj & RFj & LTj \\
\hline $\mathrm{T}$ & $17,8 \pm 7,04^{\mathrm{a}}$ & $18,7 \pm 6,82^{a}$ & $15,1 \pm 8,05^{a}$ & $18,6 \pm 7,10^{a}$ \\
\hline $\mathrm{pH}$ & $6,0 \pm 0,30^{a}$ & $6,3 \pm 0,49^{a}$ & $6,2 \pm 0,48^{a}$ & $6,4 \pm 0,50^{a}$ \\
\hline QJE & $0,17 \pm 0,023^{a}$ & $0,12 \pm 0,029^{a}$ & $0,10 \pm 0,023^{a}$ & $0,15 \pm 0,042^{a}$ \\
\hline $\mathrm{CE}$ & $133,6 \pm 55,4^{b}$ & $70,8 \pm 11,71^{a}$ & $57,6 \pm 24,81^{a}$ & $138,7 \pm 60,67$ \\
\hline
\end{tabular}

$\mathrm{ST}$ : semitendinosus ; SM : semimembranosus ; RF : rectus femoris ; LT : longissimus thoracis

T : température ; QJE : quantité de jus extractible ; CE : conductivité électrique $\mathrm{a}, \mathrm{b}$ Des lettres différentes sur une même ligne montrent des différences significatives à $\mathrm{p}<0,05$

\section{Tableau II}

Moyenne \pm écart-type des paramètres physicochimiques des muscles étudiés adultes (a)

\begin{tabular}{lrrrr} 
& STa & SMa & RFa & \multicolumn{1}{c}{ LTa } \\
\hline T & $16,0 \pm 6,19^{\text {a }}$ & $18,9 \pm 7,24^{\text {a }}$ & $17,42 \pm 6,78^{\text {a }}$ & $18,6 \pm 7,23^{\text {a }}$ \\
pH & $6,3 \pm 0,36^{\text {a }}$ & $6,3 \pm 0,37^{\text {a }}$ & $6,4 \pm 0,50^{\text {a }}$ & $6,5 \pm 0,30^{\text {a }}$ \\
QJE & $0,10 \pm 0,053^{\text {a }}$ & $0,06 \pm 0,052^{\text {a }}$ & $0,12 \pm 0,045^{\text {a }}$ & $0,08 \pm 0,039^{\text {a }}$ \\
CE & $137,9 \pm 46,43^{\text {b }}$ & $76,1 \pm 28,32^{\text {a }}$ & $57,6 \pm 24,81^{\text {a }}$ & $69,7 \pm 38,11^{\text {a }}$
\end{tabular}

ST : semitendinosus ; SM : semimembranosus ; RF : rectus femoris ; LT : longissimus thoracis

T : température ; QJE : quantité de jus extractible ; CE : conductivité électrique ${ }^{a}, b$ Des lettres différentes sur une même ligne montrent des différences significatives à $\mathrm{p}<0,05$ 


\section{Corrélations entre les mesures}

Les corrélations ont été en général positivement significatives entre les différents paramètres mesurés pour les différents muscles, avec des valeurs généralement plus élevées chez l'adulte que chez le jeune, et les corrélations n'ont pas été identiques aux deux âges (tableaux III et IV). Les corrélations les plus fortes ont été relevées entre la QJE et les autres paramètres dans la plupart des muscles, aussi bien chez le jeune que chez l'adulte. A l'inverse la température a moins été liée aux autres paramètres, surtout chez le jeune. Chez l'adulte, les relations entre paramètres ont été particulièrement fortes dans les muscles SM et LT.

\section{Cinétique de la température}

La température a été un important facteur lors de l'évolution post mortem des muscles, son influence au cours du stockage peut aboutir

\section{Tableau III}

Matrice de corrélation entre les paramètres physicochimiques du semitendinosus jeune (j)

\begin{tabular}{|c|c|c|c|c|}
\hline STj & $\mathrm{T}$ & $\mathrm{pH}$ & QJE & CE \\
\hline $\mathrm{T}$ & 1 & 0,44 & 0,16 & 0,57 \\
\hline $\mathrm{pH}$ & 0,44 & 1 & 0,65 & 0,65 \\
\hline QJE & 0,16 & 0,65 & 1 & 0,24 \\
\hline CE & 0,57 & 0,65 & 0,24 & 1 \\
\hline SMj & $\mathrm{T}$ & $\mathrm{pH}$ & QJE & CE \\
\hline $\mathrm{T}$ & 1 & 0,14 & 0,21 & 0,08 \\
\hline $\mathrm{pH}$ & 0,14 & 1 & 0,45 & 0,15 \\
\hline QJE & 0,21 & 0,45 & 1 & 0,77 \\
\hline CE & 0,08 & 0,15 & 0,77 & 1 \\
\hline RFj & $\mathrm{T}$ & $\mathrm{pH}$ & QJE & CE \\
\hline $\mathrm{T}$ & 1 & 0,89 & 0,68 & 0,40 \\
\hline $\mathrm{pH}$ & 0,89 & 1 & 0,44 & 0,34 \\
\hline QJE & 0,68 & 0,44 & 1 & 0,29 \\
\hline CE & 0,40 & 0,34 & 0,29 & 1 \\
\hline LTj & $\mathrm{T}$ & $\mathrm{pH}$ & QJE & CE \\
\hline $\mathrm{T}$ & 1 & 0,33 & 0,43 & 0,27 \\
\hline $\mathrm{pH}$ & 0,33 & 1 & 0,60 & 0,67 \\
\hline QJE & 0,43 & 0,60 & 1 & 0,68 \\
\hline CE & 0,27 & 0,67 & 0,68 & 1 \\
\hline
\end{tabular}

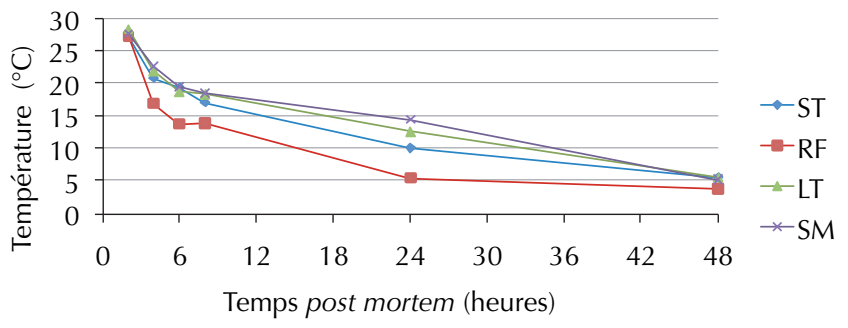

Figure 1 : évolution post mortem de la température des muscles jeunes. $S T$ : semitendinosus ; RF : rectus femoris ; $L T$ : longissimus thoracis; $S M$ : semimembranosus. à des variations importantes sur le phénomène global de la transformation du muscle en viande et, de ce fait, sur les propriétés organoleptiques finales de la viande. Pour cela, les muscles étudiés ont subi le même protocole thermique, afin que ce facteur ne soit pas à l'origine des différences pouvant exister entre eux. Le suivi de la température avait donc pour intérêt le contrôle du régime thermique des muscles et la différence de vitesse dans le transfert thermique selon le type du muscle. Les quatre muscles ont eu la même cinétique pour ce paramètre, avec une baisse rapide au cours des six premières heures, suivie d'une baisse plus lente entre $8 \mathrm{~h}$ et $48 \mathrm{~h}$ (figures 1 et 2). Le point d'arrivée a été comparable dans tous les cas, se rapprochant de la température ambiante de stockage $\left(4{ }^{\circ} \mathrm{C}\right)$ mais des différences entre les muscles ont été observées jusqu'à $24 \mathrm{~h}$. Chez le jeune, RF a eu une baisse plus rapide et plus forte de la température que les autres muscles. Au contraire, SM a eu une baisse un peu plus modérée aussi bien chez le jeune que chez l'adulte.

\section{Tableau IV}

Matrice de corrélation entre les paramètres physicochimiques du semitendinosus adulte (a)

\begin{tabular}{lllll} 
STa & T & pH & QJE & CE \\
\hline T & 1 & 0,59 & 0,38 & 0,32 \\
$\mathrm{pH}$ & 0,59 & 1 & 0,45 & 0,31 \\
QJE & 0,38 & 0,45 & 1 & 0,52 \\
$\mathrm{CE}$ & 0,32 & 0,31 & 0,52 & 1 \\
$\mathrm{SMa}$ & $\mathrm{T}$ & $\mathrm{pH}$ & $\mathrm{QJE}$ & $\mathrm{CE}$ \\
\hline $\mathrm{T}$ & 1 & 0,74 & 0,80 & 0,64 \\
$\mathrm{pH}$ & 0,74 & 1 & 0,81 & 0,74 \\
$\mathrm{QJE}$ & 0,80 & 0,81 & 1 & 0,79 \\
$\mathrm{CE}$ & 0,64 & 0,74 & 0,79 & 1 \\
$\mathrm{RFa}$ & $\mathrm{T}$ & $\mathrm{pH}$ & $\mathrm{QJE}$ & $\mathrm{CE}$ \\
\hline $\mathrm{T}$ & 1 & 0,28 & 0,34 & 0,29 \\
$\mathrm{pH}$ & 0,28 & 1 & 0,37 & 0,38 \\
QJE & 0,34 & 0,37 & 1 & 0,89 \\
$\mathrm{CE}$ & 0,29 & 0,38 & 0,89 & 1 \\
& & & & \\
$\mathrm{LTa}$ & $\mathrm{T}$ & $\mathrm{pH}$ & $\mathrm{QJE}$ & $\mathrm{CE}$ \\
\hline $\mathrm{T}$ & 1 & 0,60 & 0,70 & 0,66 \\
$\mathrm{pH}$ & 0,60 & 1 & 0,54 & 0,50 \\
QJE & 0,70 & 0,54 & 1 & 0,68 \\
$\mathrm{CE}$ & 0,66 & 0,50 & 0,68 & 1 \\
& & & &
\end{tabular}

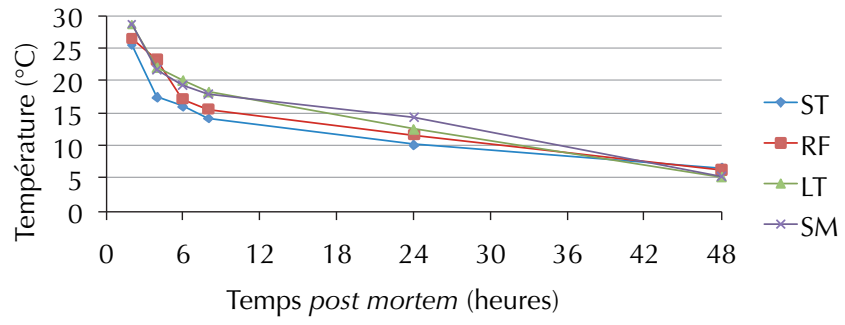

Figure 2 : évolution post mortem de la température des muscles jeunes. ST : semitendinosus ; RF : rectus femoris ; $L T$ : longissimus thoracis; $S M$ : semimembranosus. 


\section{Cinétique du pH}

L'évolution du pH post mortem a suivi le même profil, se caractérisant par une chute rapide pendant les huit premières heures, suivie par un ralentissement (figures 3 et 4). Les valeurs initiales du pH des muscles d'animaux ont été environ de 6,8 en moyenne, avec des valeurs plus variables chez le jeune $(6,5$ pour $\mathrm{SM} v s$ 7,1 pour LT). Une légère baisse du $\mathrm{pH}$ entre $24 \mathrm{~h}$ et $48 \mathrm{~h}$ a été observée dans certains muscles (ST et RF chez le jeune, ST chez l'adulte). Chez le jeune, le muscle RF a eu un pH stable entre $8 \mathrm{~h}$ et $24 \mathrm{~h}$, puis une baisse à $48 \mathrm{~h}$, menant à la plus faible valeur enregistrée $(5,2)$. Chez l'adulte au contraire le $\mathrm{pH}$ de RF a été plus stable dès $8 \mathrm{~h}$ et a terminé beaucoup plus haut que le $\mathrm{pH}$ des autres muscles $(6,3)$.

\section{Cinétique de la capacité de rétention d'eau tissulaire}

La quantité d'eau extractible a augmenté au cours de la période post mortem ce qui signifie une diminution de la capacité de rétention d'eau des protéines. L'évolution de la capacité de rétention d'eau a été biphasique, se caractérisant par une augmentation rapide durant les huit premières heures qui ont suivi l'abattage (figures 5 et 6). Les valeurs initiales de la QJE ont été plus fortes chez le jeune que chez l'adulte $(9,3$ vs $3,5 \mathrm{~g} / \mathrm{g}$ de muscle). Des différences importantes ont été observées entre les muscles, dès les premières heures, et se sont accentuées à $24 \mathrm{~h}$, puis à $48 \mathrm{~h}$. Chez le jeune, une grande différence a été observée entre les muscles, avec des pertes minimales pour RF et maximales pour LT. Chez l'adulte, c'est ST qui a eu les valeurs les plus fortes à $48 \mathrm{~h}$, tandis que les trois autres muscles ont été comparables. RF a eu une évolution particulière puisque sa QJE a été la plus élevée dans toute la première phase de la maturation, jusqu'à $24 \mathrm{~h}$, avant de baisser et devenir la valeur la plus faible à $48 \mathrm{~h}$.

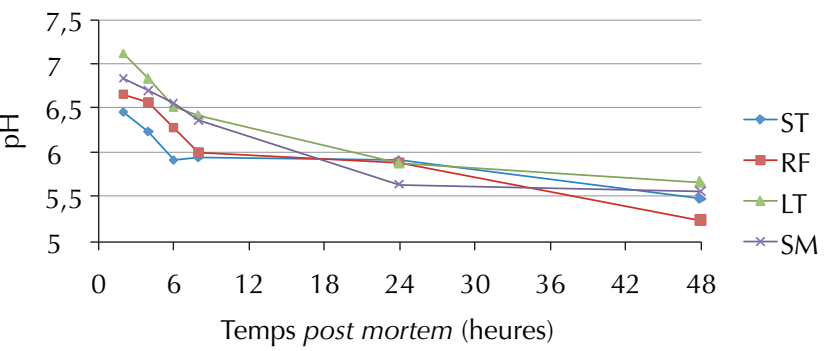

Figure 3 : évolution post mortem $d u p H$ des muscles jeunes. $S T$ : semitendinosus ; RF : rectus femoris ; $L T$ : longissimus thoracis ; $S M$ : semimembranosus.

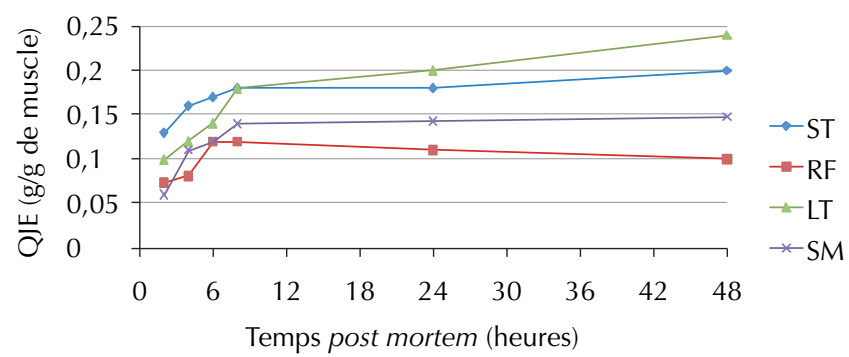

Figure 5 : évolution post mortem de la quantité du jus relarguée par les muscles jeunes. QJE : Quantité du jus rélargué ; $S T$ : semitendinosus ; RF : rectus femoris ; $L T$ : longissimus thoracis; $S M$ : semimembranosus.

\section{Cinétique de la conductivité électrique}

La conductivité électrique est un paramètre qui nous a renseignés sur l'évolution de la totalité des ions dans le tissu au cours du post mortem. Chez le jeune, les muscles ST et LT ont eu des valeurs initiales très fortes (respectivement 246 et $274 \mu \mathrm{S} / \mathrm{cm} / \mathrm{g}$ à $2 \mathrm{~h}$ ) comparativement à $\mathrm{SM}$ et surtout $\mathrm{RF}(28 \mu \mathrm{S} / \mathrm{cm} / \mathrm{g})$ (figure 7$)$. Les valeurs ont ensuite convergé avec une très forte baisse à $4 \mathrm{~h}$ pour ST et LT, et une augmentation pour RF, aboutissant vers $8 \mathrm{~h}$ à des valeurs plus rapprochées (75 à $121 \mu \mathrm{S} / \mathrm{cm} / \mathrm{g})$. Les valeurs ont ensuite été plus stables jusqu'à $48 \mathrm{~h}$ avec toutefois une baisse de la CE pour SM entre $8 \mathrm{~h}$ et $24 \mathrm{~h}$, et pour ST et RF entre $24 \mathrm{~h}$ et $48 \mathrm{~h}$. Au final, la CE à $48 \mathrm{~h}$ a été la plus élevée dans LT $(117 \mu \mathrm{S} / \mathrm{cm} / \mathrm{g})$.

Chez l'adulte, la CE n'a pas montré le même type de profil, en l'absence de valeurs initiales élevées. Elle a augmenté entre $2 \mathrm{~h}$ et $8 \mathrm{~h}$ pour tous les muscles : plus précocement (dès $4 \mathrm{~h}$ ) et plus fortement pour ST, plus tardivement pour SM et LT (figure 8). A $8 \mathrm{~h}$ une assez grande dispersion de la CE a été présente, avec les valeurs les plus fortes pour ST $(174 \mu \mathrm{S} / \mathrm{cm} / \mathrm{g})$ et les plus faibles pour RF $(75 \mu \mathrm{S} / \mathrm{cm} / \mathrm{g})$. Les valeurs ont ensuite été relativement stables entre $8 \mathrm{~h}$ et $48 \mathrm{~h}$, avec seulement une baisse notable pour $\mathrm{RF}$ de $24 \mathrm{~h}$ à $48 \mathrm{~h}$.

\section{DISCUSSION}

La température de stockage des muscles a été un facteur très important pour le développement des caractéristiques organoleptiques de la viande (7). Dans notre étude, les muscles ont subi un régime thermique identique, afin d'éliminer son effet sur l'évolution de leur maturation. L'étude des caractéristiques physicochimiques de la viande de dromadaire conservée à $4{ }^{\circ} \mathrm{C}$ a fait apparaître pour les quatre types de muscles étudiés provenant d'animaux jeunes ou adultes une chute de la température au cœur

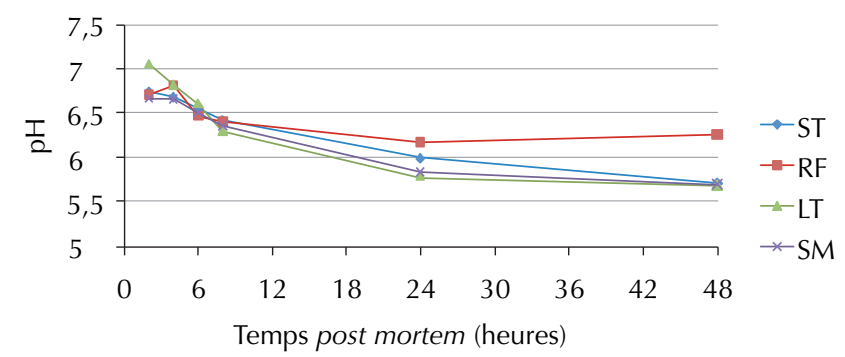

Figure 4 : évolution post mortem $d u p H$ des muscles jeunes. $S T$ : semitendinosus ; $R F$ : rectus femoris ; $L T$ : longissimus thoracis ; $S M$ : semimembranosus.

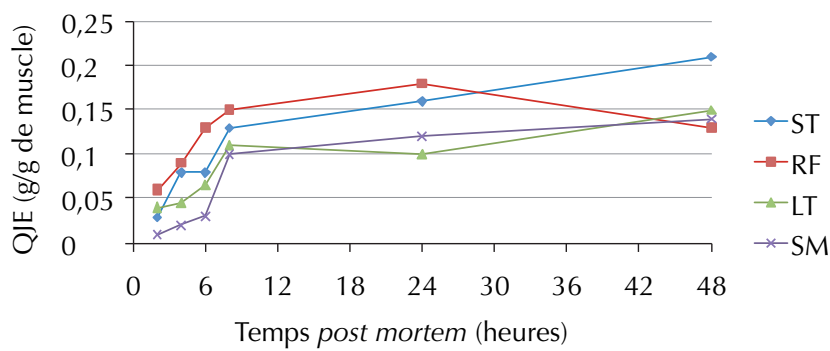

Figure 6 : évolution post mortem de la quantité du jus relarguée par les muscles jeunes. QJE : Quantité du jus rélargué ; $S T$ : semitendinosus ; RF : rectus femoris ; $L T$ : longissimus thoracis ; $S M$ : semimembranosus. 


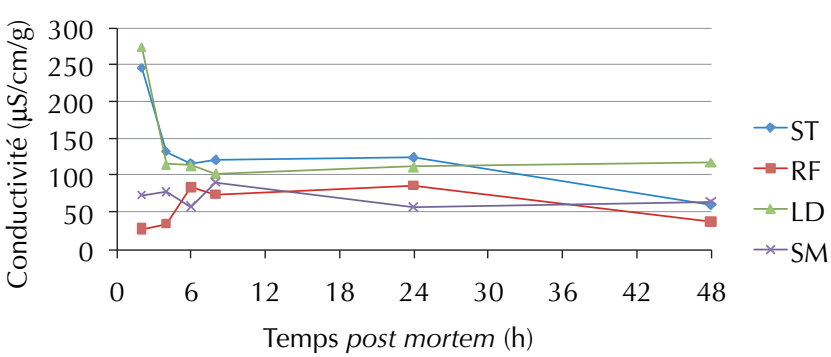

Figure 7 : évolution post mortem de la conductivité électrique muscles jeunes. CE : Conductivité électrique ; ST : semitendinosus ; $R F$ : rectus femoris ; $L T$ : longissimus thoracis ; $S M$ : semimembranosus.

du muscle, avec des différences enregistrées liées à la variabilité de la morphologie des carcasses et à la composition intrinsèque de chaque muscle selon sa teneur en matières grasses (21). Ces différences peuvent être dues à l'influence de la température de l'animal lors de l'abattage, la température de l'environnement (saison de l'année) et la résistance du muscle au froid (son contenu en graisse).

Dans les deux heures qui suivent l'abattage, l'énergie nécessaire à la survie des cellules est fournie par l'utilisation de ses réserves en phosphocréatines (12). Après l'épuisement de ces dernières, l'énergie est produite par la dégradation du glycogène en anaérobiose par glycolyse produisant ainsi de l'acide lactique qui s'accumule par la suite à l'intérieur des cellules, causant ainsi une chute du pH des muscles. L'acidification est proportionnelle à l'efficacité du système glycolytique et aux réserves en glycogène (4). La baisse du pH joue un rôle important dans le devenir de la viande car pour un $\mathrm{pH}$ de 5,5 à 5,8, l'activation des enzymes protéolytiques influent fortement sur la qualité organoleptique de la viande (22) au cours de la maturation. Selon, Ngoka et coll. (15), la chute du pH n'est pas en relation avec l'âge ni avec le sexe de l'animal, mais plutôt avec l'accumulation de l'acide lactique, ce qui engendre une acidification progressive des muscles, celle-ci se poursuivant jusqu'à l'arrêt des réactions biochimiques anaérobies. Le stress subi par l'animal avant l'abattage contribue aussi à l'évolution de ce paramètre (8).

Selon Becila (1), l'évolution des paramètres biologiques est influencée par l'état de l'animal. L'augmentation de la quantité d'eau relarguée au cours du post mortem enregistrée dans notre étude peut être la conséquence de la chute du pH influençant le pouvoir des myofibrilles à retenir l'eau et par conséquent la capacité de rétention d'eau de la viande (16). Selon Boakye et Mihal (2), la capacité de rétention d'eau des muscles diminue rapidement quand la vitesse de chute du $\mathrm{pH}$ augmente. Ce paramètre intervient dans le phénomène de transformation des muscles en viande et renseigne sur leur jutosité et tendreté finales (26). Ouali et coll. (18) rapportent pour les muscles de bovins à $48 \mathrm{~h}$ après abattage des valeurs légèrement inférieures $(0,250 \mathrm{~g} / \mathrm{g}$ de muscle). Cela peut s'expliquer par le fait que la viande de dromadaire contient plus de jus extractible que les autres ruminants à cause de la contenance plus faible en gras (5). Aussi, la protéolyse post mortem provoque des changements irréversibles dans l'espace entre les myofibrilles, causant une augmentation de la quantité du jus libéré (3). D'après nos résultats, on note une augmentation des valeurs de ce paramètre jusqu'à 8 heures, suivie d'une phase de stabilité pour tous les muscles étudiés à l'exception des deux muscles jeunes ST et SM dont le profil commence par une diminution, suivie par une stabilité. Le profil d'augmentation de ce paramètre a été signalé par Troy (24) sur le muscle bovin, et par Veiseth et coll. (25) sur le muscle ovin.

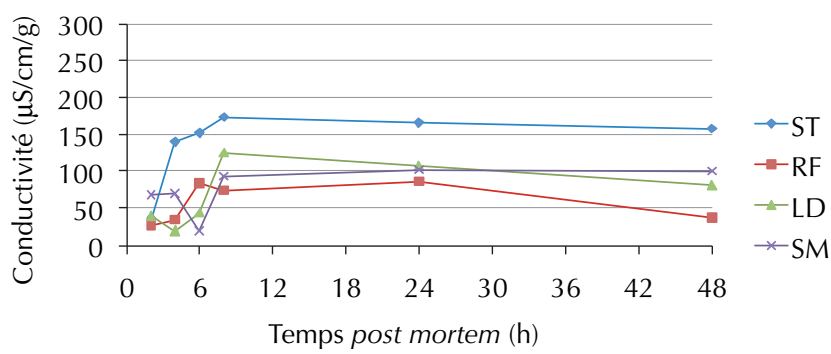

Figure 8 : évolution post mortem de la conductivité électrique muscles jeunes. CE : Conductivité électrique ; ST : semitendinosus ; $R F$ : rectus femoris ; $L T$ : longissimus thoracis ; $S M$ : semimembranosus.

\section{CONCLUSION}

L'étude de la cinétique de quelques paramètres physico-chimiques de quatre muscles au post mortem chez des camelins de race Sahraoui, jeunes et adultes, a mis en évidence la chute de la température et du pH, alors que les valeurs de la quantité de jus relarguée par le tissu et la conductivité électrique ont augmenté au cours des 48 heures qui ont suivi la saignée. On note un effet non significatif de l'âge à l'abattage sur le $\mathrm{pH}$ et la capacité de rétention d'eau des protéines myofibrillaires.

\section{BIBLIOGRAPHIE}

1. BECILA S., 2009. Marqueurs biologiques de la qualité de la viande ovine et caractérisation de la mise en place de l'apoptose. Thèse Doct. Université de Constantine, Algérie, p. 28-30.

2. BOAKYE K., MIHAL G.S., 1993. Change in $\mathrm{pH}$ and water-holding proprieties of Longissimus dorsi muscle during beef ageing. Meat Sci., 34: 335-349. DOI: 10.1016/0309-1740(93)90082-S

3. BOND J.J., WARNER R.D., 2007. Ion distribution and protein proteolysis affect water holding capacity of Longissimus thoracis and lumborum in meat of lamb subjected to ante mortem exercise. Meat Sci., 75: 406-414. DOI: 10.1016/j.meatsci.2006.08.005

4. BOUDJELLAL A., BECILA S., COULIS G., HERRERA-MENDEZ C., AUBRY L., LEPETIT J., HARHOURA K., SENTANDREU M.A., AIT AMAR H., OUALI A., 2008. Is the $\mathrm{pH}$ drop profile curvilinear and either monophasic or polyphasic? Consequences on the ultimate bovine meat texture, Afric. J. Agric. Res., 3: 195-204.

5. CRISTOFANELLI S., ANTONINI T., TORRES D., POLIDORI P., RENIERI C., 2004. Meat and carcass quality from peruvian Ilama (Lama glama) and alpaca (Lama pacos). Meat Sci., 66: 589-593. DOI: 10.1016/S0309. 1740(03)00174-8

6. CULIOLI J., 1999. La qualité de la viande bovine : aspects biologiques et technologiques de la gestion de la tendreté. Bull. Acad. Vét. Fr., 72 : 25-46.

7. DEVINE C.E., PAYNE S.R., PEACHEY B.M., LOWE T.E., INGRAM J.R. COOK C.J., 2002. High and low rigor temperature effects on sheep meat tenderness and ageing. Meat Sci., 60: 141-146. DOI: 10.1016/S0309. 1740(01)00115-2

8. El RAMMOUZ R., 2005. Etude des changements biochimiques post mortem dans le muscle des volailles. Contribution au déterminisme de l'amplitude de la diminution du $\mathrm{pH}$. Thèse Doct., Institut national polytechnique, Toulouse, France, $138 \mathrm{p}$.

9. FAYE B., ABDELHADI O., RAIYMBEK G., KADIM I., HOCQUETTE J.F., 2013. La production de viande de chameau : état des connaissances, situation actuelle et perspectives. Prod. Anim., 26 : 247-258.

10. GEAY Y., BAUCHART D., HOCQUETTE J.-F., CULIOLI J., 2002. Valeur diététique et qualités sensorielles des viandes des ruminants. Incidence de I'alimentation des animaux. Prod. Anim., 15 : 37-52.

11. HOCQUETTE J.F., BOTREAU R., PICARD B., JACQUET A. PETHICK D.W., SCOLLAN N.D., 2012. Opportunities for predicting and manipulating beef quality. Meat Sci., 92: 197-209. DOI: 10.1016/j. meatsci.2012.04.007 
12. HONIKEL K.O., 2009. Moisture and water-holding-capacity. In: Nollet L.M.L., Toldra F., Eds, Handbook of muscle foods analysis. Boca Raton, FL, USA, CRC Press, p. 315-332.

13. JIANG S.T., 2000. Effect of proteinases on the meat texture and seafood quality. Food Sci. Agric. Chem., 2: 55-74.

14. KOOHMARAIE M., 1994. Muscle proteinases and meat ageing. Meat Sci., 36: 93-104. DOI: 10.1016/0309-1740(94)90036-1

15. NGOKA D.A., FRONING G.W., LOWRY S.R., BABJI A.S., 1982 Effect of sex, age, preslaughter factors and holding conditions on the quality characteristics and chemical composition of turkey breast muscles. Poult. Sci., 61: 1996-2003. DOI: 10.3382/ps.0611996

16. OFFER G., 1983. On mechanism of water-holding in meat. The swelling and shrinking of myofibrils. Meat Sci., 8: 245-281. DOI: 10.1016/0309-1740(83)90013-X

17. OUALI A., OBLED A., DEVAl C., VALIN C., 1987. Comparative action of cathepsins $D, B, H, L$ and of new lysosomal cysteine proteinase on rabbit myofibrils. Meat Sci., 19: 83-100. DOI : 10.1016/03091740(87)90014-3

18. OUALI A., VIGNON X., BONNET M., 1991. Osmotic pressure in post mortem bovine muscles: factors of variation and possible causative agents. In: Proc. 37th. Int. Congr. Meat Science and Technology, Kulmbach, Germany, 1-6 Sept. 1991, Vol. I, p. 452-456.

19. OULD EI HADJ M.D., BOUZGAG B., BOURAS A., MOUSSAOU S., 2002. Etude comparative de quelques caractéristiques chimiques et physico-chimiques de la viande du dromadaire chez des individus du type "sahraoui », différents âges. In : Première journée sur la recherche cameline, Université de Ouargla, Algérie. Rech. Agron. INRA, 10 : 95102.

\section{Summary}

Benaissa A., Ould El Hadj-Khelil A., Adamou A., Babelhadj B., Mehirig M., Boufaghes B., Attoussi M., Samili H., Becila S. Quality of camel meat in the slaughterhouse of Ouargla in Algeria. I. Some physical and chemical characteristics of meat during ageing

The physicochemical parameters of four muscles (semitendinosus [ST], rectus femoris [RF] longissimus thoracis [LT] and semimembranosus $[\mathrm{SM}]$ ), stored at $4^{\circ} \mathrm{C}$, were measured during ageing in male camels (10 young aged three to four years, and 10 adults over five years) of Sahrawi breed. A decrease in postmortem temperature reached a minimum of $5.7 \pm 0.20^{\circ} \mathrm{C}$ (LT) in adults, whereas the minimum value in the young was lower with $3.9 \pm 0.68^{\circ} \mathrm{C}(\mathrm{ST})$. The $\mathrm{pH}$ of the studied muscles decreased during the postmortem period. The minimum values ranged from $5.2 \pm 1.11$ (RF) to $5.7 \pm 0.02$ (LT) in the young, and from $5.6 \pm 0.05(\mathrm{SM})$ to $6.3 \pm 053$ (ST) in adults. The amount of extractable water increased for the four muscles in both age groups. The maximum values were $0.21 \pm 0.040 \mathrm{~g}$ per gram of RF muscle in the young, and $0.21 \pm 0.009 \mathrm{~g}$ per gram of RF muscle in adults. This meant that the water retention capacity of the different muscles decreased during the postmortem period. The electrical conductivity did not follow the same pattern for all the studied muscles. A slight increase in this parameter was detected over time in LT $(117.3 \pm 0.30$ $\mu \mathrm{S} / \mathrm{cm} / \mathrm{g})$ in the young, and in ST $(158.3 \pm 0.14 \mu \mathrm{S} / \mathrm{cm} / \mathrm{g})$ in the adults.

Keywords: Camelus dromedarius - Camel meat - Muscle Maturation of products - Chemicophysical property - $\mathrm{pH}-$ Algeria.
20. PICARD B., JURIE C., CASSAR M., HOCQUETTE J.F., 2002. Typologie et myogenèse des fibres musculaires chez le bovin. Prod. Anim., 16 : 125-131.

21. SMULDERS F.J.M., VAN LAAK R.L.J., EIKELENBOOM G., 1991. Muscle and meat quality: biological basis processing preparation. In: Smulders F.M.J., Ed., The European meat industry in the 1990's advanced technologies product quality and consumer acceptability. Utrecht, Netherlands, ECCEAMST/Audet, p. 121-159.

22. STARON T., 1981. L'alimentation humaine. Vol. 1 : Contribution à l'étude des constituants alimentaires et des aliments. Paris, France, Apria, $163 \mathrm{p}$.

23. TROUT G.R., 1988. Techniques for measuring water-holding capacity in muscle foods: a review of methodology. Meat Sci., 23: 235-252. DOI: 10.1016/0309-1740(88)90009-5

24. TROY D.J., 1999. Biochemical and physical indicators of beef quality. Dublin, Ireland, The National Food Center, 36 p. (Res. Rep. No 13)

25. VEISETH E., SHACHELFORD S.D., WHEELER T., KOOHMARAIE M., 2004. Indicators of tenderisation are detectable by $12 \mathrm{~h}$ post mortem in ovine longissimus. J. Anim. Sci., 82: 1428-1436.

26. ZAMORA F., 1997. Variabilité biologique de I'attendrissage de la viande bovine : prédiction en fonction du facteur animal et du facteur type de muscle. Thèse Doct., Université Blaise Pascal, Clermont-Ferrand, France, $175 \mathrm{p}$.

27. ZAMORA F., DEBITON E., LEPETIT J., LEBERT A., DRANSFIELD E., OUALI A., 1996. Predicting variability of ageing and toughness in beef. Longissimus lumborum and thoracis. Meat Sci., 43: 321-333. DOI: 10.1016/S0309-1740(96)00020-4

Accepted 20 August 2015; Online publication 30 September 2015

\section{Resumen}

Benaissa A., Ould El Hadj-Khelil A., Adamou A., Babelhadj B., Mehirig M., Boufaghes B., Attoussi M., Samili H., Becila S. Calidad de la carne de dromedario en los mataderos de Ouargla en Argelia. I. Algunas características físico-químicas de la carne durante la maduración

Los parámetros físico-químicos durante la maduración de cuatro músculos (semitendinoso [ST], rectus femoris [RF], longissimus thoracis [LT] y semimembranoso [SM]), conservados a $+4^{\circ} \mathrm{C}$, fueron medidos en dromedarios macho (10 jóvenes de tres a cuatro años y 10 adultos de más de cinco años) de raza Saharaoui. Una disminución de la temperatura post mortem alcanzó un valor mínimo de 5,7 $\pm 0,20^{\circ} \mathrm{C}$ (LT) en los adultos, mientras que el valor mínimo registrado en los jóvenes fue más bajo con $3,9 \pm 0,68^{\circ} \mathrm{C}(\mathrm{ST})$. $\mathrm{El} \mathrm{pH}$ de los músculos estudiados disminuyó durante el periodo post mortem. Los valores mínimos registrados variaron entre 5,2 $\pm 1,11$ (RF) y 5,7 $\pm 0,02(\mathrm{LT})$ en los camellejos, y de 5,6 $\pm 0,05(\mathrm{SM})$ y $6,3 \pm 0,53$ (ST) en los adultos. La cantidad de agua extraíble aumentó para los cuatro músculos y en los dos grupos etáreos. Los valores máximos obtenidos fueron de 0,21 $\pm 0,040$ g por gramo de músculo RF joven y de 0,21 $\pm 0,009$ g por gramo de músculo RF adulto. Esto significa que la capacidad de retención de agua de los diferentes músculos disminuyó con el curso del tiempo post mortem. La conductividad eléctrica no siguió el mismo perfil para todos los músculos estudiados. Una ligera disminución de este parámetro con el curso del tiempo fue perceptible para el músculo LT $(117,3$ $\pm 0,30 \mu \mathrm{S} / \mathrm{cm} / \mathrm{g})$ en los jóvenes, y ST $(158,3 \pm 0,14 \mu \mathrm{S} / \mathrm{cm} / \mathrm{g})$ en los adultos.

Palabras clave: Camelus dromedarius - Carne de camello Músculo - Maduración de productos - Propiedad fisicoquímica - $\mathrm{pH}$ - Argelia. 